\title{
Biological Pathway Extension Using Microarray Gene Expression Data
}

\author{
Tae Su Chung ${ }^{1,2 \dagger}$, Jihun $\mathrm{Kim}^{1 \dagger}$, Keewon $\mathrm{Kim}^{1}$ \\ and Ju Han $\mathrm{Kim}^{1,2 *}$
}

${ }^{1}$ Seoul National University Biomedical Informatics (SNUBI), Seoul National University College of Medicine, Seoul 110-799, Korea, ${ }^{2}$ Human Genome Research Institute, Seoul National University College of Medicine, Seoul 110-799, Korea

\begin{abstract}
Biological pathways are known as collections of knowledge of certain biological processes. Although knowledge about a pathway is quite significant to further analysis, it covers only tiny portion of genes that exists. In this paper, we suggest a model to extend each individual pathway using a microarray expression data based on the known knowledge about the pathway. We take the Rosetta compendium dataset to extend pathways of Saccharomyces cerevisiae obtained from KEGG (Kyoto Encyclopedia of genes and genomes) database. Before applying our model, we verify the underlying assumption that microarray data reflect the interactive knowledge from pathway, and we evaluate our scoring system by introducing performance function. In the last step, we validate proposed candidates with the help of another type of biological information. We introduced a pathway extending model using its intrinsic structure and microarray expression data. The model provides the suitable candidate genes for each single biological pathway to extend it.
\end{abstract}

Keywords: biological pathway, pathway extension, microarray gene expression

\section{Introduction}

Biological pathways can be regarded as a collection of known relations or reactions between biological objects (i.e. genes or gene products). But the knowledge about biological pathway is not complete and insufficient to cover the whole number of genes or gene products. In case of human, for example, about 3,000 genes are

\footnotetext{
*Corresponding author: E-mail juhan@snu.ac.kr

${ }^{\dagger}$ Contributed equally

Tel +82-2-740-8320, Fax +82-2-762-3063

Accepted 19 October 2008
}

covered by the major biological pathway databases. The rest with the relation with other genes remains to be explored. We view biological pathways as a set of biological knowledge that should be expanded by using experimental data like DNA microarray gene expression data.

Recently, microarray gene expression datasets are incessantly cumulated with the aid of recent technological advances. Analyzing these datasets makes it possible to measure the expressions of tens of thousands of genes simultaneously under various experimental conditions. In this paper, we propose a model, PathPlus, to determine genes for biological pathway extension by combining the knowledge about biological pathways and the experimental data from DNA microarray experiments.

The most significant feature of PathPlus is that it uses established knowledge (i,e biological pathway) as a template to expand the knowledge. Previous studies try to directly infer biological networks (or pathways) from gene expression data by various computational or statistical means without using prior knowledge (or template). To induce networks of genes or proteins, for example, that might conjecture biological pathways from large-scale data, some construct bayesian networks from gene expression data or infer functional protein complexes from protein interaction data (Friedman et al., 2000). Others combine various genomic data to infer biological networks without using prior knowledge about biological interaction. In fact, there are a few recent works trying to reconstruct biological relations based on prior knowledge (Yamanishi et al., 2004; Kharchenko et al., 2004). Yamanishi et al. uses kernel method to predict new gene-to-gene interaction within metabolic pathway and bases it on known pathway knowledge by adopting supervised approach. The work of Kharchenko et al. compares established metabolic network with expression profiles to find genes that can complete a metabolic pathway with some participants missed. While the methods are good in finding missing genes, they do not suggest possible new members (or genes) for the given biological pathway for pathway extension.

We first observe that a biological pathway contains highly verified information but covers only a small fraction of genes, while microarray data provide noisy experimental data but covers the whole genome. The essence of PathPlus approach is to determine candidate genes that are highly likely to be related to a given pathway by combining microarray gene expression data 


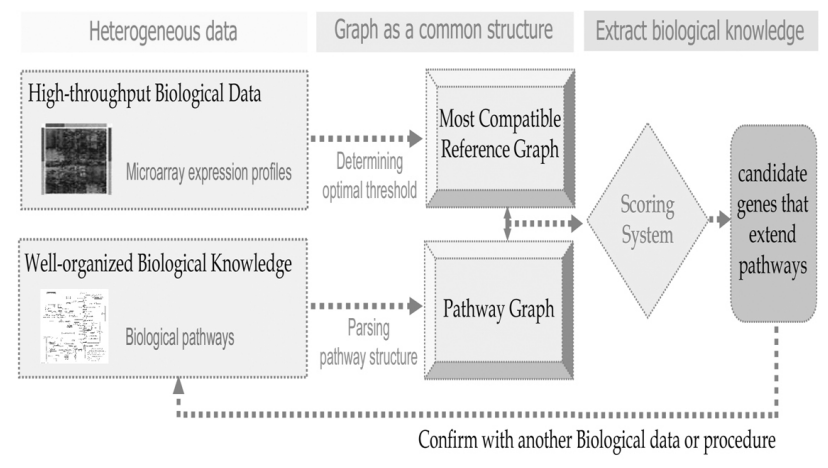

Fig. 1. Overview of PathPlus. To combine biological pathway and microarray gene expression data, that have different data structures, we transform both into graphs, pathway graphs and reference graphs. Applying a scoring system, we obtain a set of candidate genes with high scores for each pathway. The set is verified by using another knowledge resource, the Gene Ontology.

and the known knowledge about the biological pathway (Fig. 1).

To combine biological pathways and microarray datasets, that are very different in data structure, we transform both into a common template structure, a graph. We transform each pathway into a graph whose nodes correspond to genes or gene products and edges to biological relations between them. Then, we extract from microarray data a reference graph having the largest structural similarity with the given pathway graph by optimizing the parameters of graph construction. PathPlus provides a score measuring the likelihood of a gene to be related to a pathway using the structure of the reference graph. The scoring system is based on the centrality measures introduced by the social network analysis (Bogatti et al., 1997; Faust, 1997).

Finally, we obtain sets of candidate genes with high scores for biological pathways and evaluate the results by verifying the functions of the candidate genes using another heterogeneous data, the Gene Ontology (GO), with promising results. PathPlus approach may contribute to establish methodological concepts in knowledge extension and to give insights into biologically untapped territories.

\section{Methods}

To combine the two different data types, biological pathways and microarray gene-expression data, PathPlus use graph as the common template for analysis. A graph consists of a set of nodes and a set of edges. Nodes represent genes (or gene products) and edges gene-to-gene interactions in the present study.

\section{Graph construction from biological pathway}

For the purpose of illustration in the present study, we use pathways and gene expression profiles of Saccharomyces cerevisiae. KEGG (Kyoto Encyclopedia of genes and genomes) database (Kanehisa, 1996) provides 88 biological pathways for Saccharomyces cerevisiae. We extracted 43 pathways by removing those containing less than 12 genes to avoid the perturbation caused by the scarcity of basis knowledge. KEGG relations are divided into three categories: EC relation, PP relations and GE relation. EC relation stands for relations between two genes whose protein products share the same metabolite in a metabolic pathway. When two proteins interact directly, genes coding them are said to have PP relation. GE relation means that one gene or its product regulates the expression of the other gene. In addition, we define co-member relation that refers to the genes assigned to the same function in a pathway. To construct a pathway graph from this information, we make a node for each gene and link a pair of nodes when they are assigned one of the relations listed above.

\section{Graph construction from microarray expression data}

As a source of microarray expression data, we use the Rosetta compendium dataset (Hughes et al., 2000), which is hitherto the most systematic approach to profile yeast genes. The dataset is consisted of 300 microarray experiment results, which contain 287 diverse gene mutations and 13 chemical treatments. They all cover 6,153 genes in each microarray data. The log-expression ratio values are used as entries of expression matrix, and these values are normalized so that mean and standard deviation of each column are 0 and 1 , respectively.

The whole genes in the microarray dataset make nodes in reference graph of the pathway graph for each pathway we will concern. We here present two different methods to construct graph from the microarray dataset. Each method needs thresholds in constructing graphs and we will give criteria to optimize threshold so that the resulting graph reflects the biological knowledge in some sense.

Method of linking co-DEGs: The process of identifying DEGs (differentially expressed genes) is one of the major steps of analyzing gene expression data under the assumption that the expression data are modeled to find significant changes in gene expression levels between treatment conditions and control conditions.

Many reasonable procedures and statistical methods 
for optimizing threshold to determining DEGs are introduced and applied to further analysis (lyer et al., 1999; Tusher et al., 2001; Dudoit et al., 2002). Linking co-DEGs methods constructs a graph by linking two genes that are co-DEGs, i.e., they are DEGs under same experimental condition. The method was applied to the microarray expression dataset with various threshold values.

Method of linking similarly expressed genes: This method is the way of determining edges based on expression similarity. In this paper, the absolute value of Pearson correlation coefficient is taken as similarity system. When a similarity of a pair of genes exceeds a given threshold, these two genes are linked in this method.

\section{The most compatible reference graph of path- way graph}

Among all possible values of threshold, single value is to be determined so that the compatibility between pathway graph and reference graph can be maximized. The compatibility between two graph structures is designed to indicate the measurement by which two graph structures are similar. Here we will introduce the notion of compatibility between two graphs, in the general context of graph theory. The geodesic distance $d(g, h ; G)$ between nodes $g$ and $h$ is defied by the length of shortest path from $g$ to $h$ in the graph $G$. This distance rep- resents the global structure of graph (Chartrand et al., 1988). If two graphs $G 1$ and $G 2$ are constructed on the same set of nodes, then the geodesic distance of two graphs can be easily defined by the average of differences of all geodesic distances of all pairs of nodes in each graph, i.e.

$$
\operatorname{dist}\left(G_{1}, G_{2}\right)=\frac{\sum\left|d\left(g, h ; G_{1}\right)-d\left(g, h ; G_{2}\right)\right|}{n(n-1) / 2}
$$

where the summation is taken over all (unordered) pairs of nodes and $n$ is the number of common set of nodes. We here note that it is symmetric and satisfies triangle inequality (Chartrand et al., 1988). Now let $G_{P}$ be the pathway graph and $G_{M}=G_{M}(q)$ is a (reference) graph constructed by microarray data with threshold q using certain graph construction method. Then the compatibility $\operatorname{Comp}\left(G_{P}, G_{M}\right)$ is obtained by

$$
\operatorname{Comp}\left(G_{P}, G_{M}\right)=1-\operatorname{dist}\left(G_{P}, G_{M} \mid G_{P}\right) /(n-1)
$$

Here $n$ is the number of genes in $G_{P}$, and $G_{M} \mid G_{P}$ is the relative subgraph of $G_{M}$ to $G_{P}$. Since the pathway graph $G_{P}$ contains only small subset of genes that are described in graph $G_{M}$, it is natural to compare $G_{P}$ and subgraph of $G_{M}$. It is clear that the compatibility lies between 0 and 1 and it becomes 1 only when $G_{M}$ includes exactly same suture of $G_{P}$. The most compatible reference graph of $G_{P}$ is the graph $G_{M}$ which maximize the compatibility with $G_{P}$. Simple greedy algorithm is used to find such optimal threshold for each pathway. It is
(A)
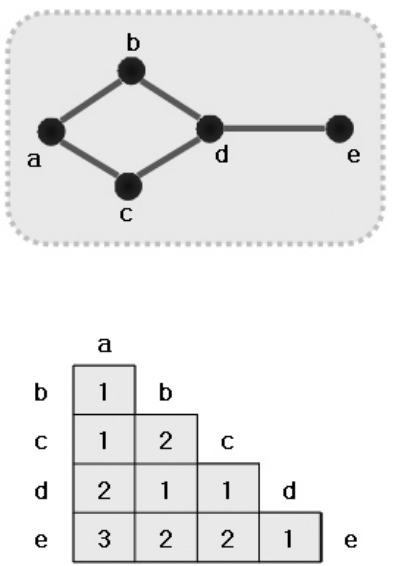

(B)
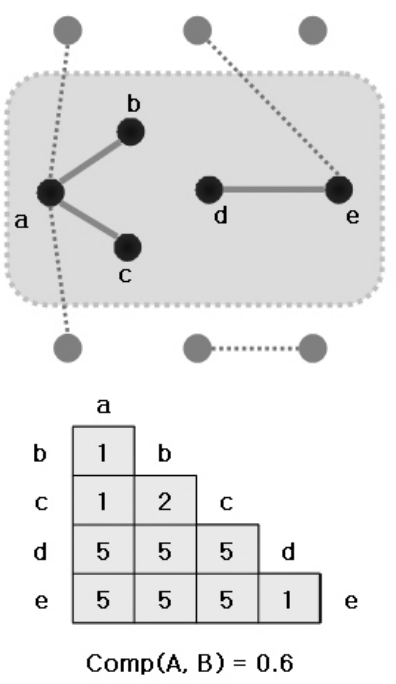

(C)
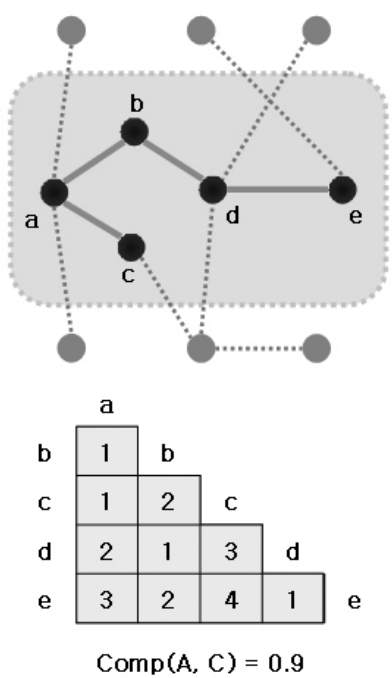

(D)
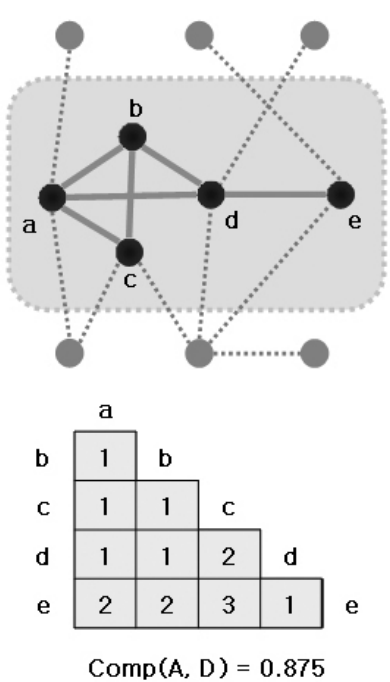

Fig. 2. Compatibilities between pathway graph and various reference graphs. When a pathway graph is given at $(A)$, various reference graphs can be compared to it according to different parameters ((B), (C) and (D)). Triangular tables represent geodesic distances of gene pairs in pathway graph (A) and reference graphs (B)-(D) which are restricted to genes in the pathway, respectively. In this figure, $(C)$ is determined to be the most compatible reference graph of $(A)$. 
worthy to note that different optimal threshold may used for extending different pathways.

The effect of threshold optimization on compatibility is illustrated in Fig. 2. When a pathway graph is given in (A), various reference graphs can be compared to it according to different parameters ((B), (C) and (D)). In this figure, $(C)$ is determined to be the most compatible reference graph of $(A)$.

\section{Scoring system}

With the most compatible reference graph of a pathway graph, we suggest a gene list supposed to be related to the given pathway by investigating scoring system which is designed to assign a higher score on a gene that is more likely to be related to the pathway. We invent scoring system based on the idea of centrality measures introduced by social network analysis (Bogatti et al., 1997). Social network analysis has its strength in reflecting the structure of a graph being observed, though it does not directly represent quantitative relations between two nodes. Because many biological networks are known to work in on-off manner, we suggest that social network analysis would be a suitable method to analyze biological networks. Here in this paper, we make our scoring system by adopting closeness centrality and betweenness centrality from social network analysis (Bogatti et al., 1997; Faust, 1997). The normalized betweenness centrality $C_{b e t}(g)$ and normalized closeness centrality $C_{c l o}(g)$ of a node $g$ are defined by

$$
C_{\text {bet }}(g)=\frac{1}{(n-1)(n-2) / 2} \sum_{\substack{x \neq 0 \\ y \neq g}} \frac{\operatorname{path}(x, y ; g)}{\operatorname{path}(x, y)}
$$

and

$$
C_{c l o}(g)=\frac{n-1}{\sum_{x \neq g} d(g, x)}
$$

Here $n$ is the number of nodes in the graph, path ( $x$, $y$ ) is the number of all shortest paths from $x$ to $y$, and path $(x, y ; g)$ is the number of shortest paths from $x$ and $y$, which pass through $g$. In the definition of closeness centrality, the geodesic distance $d(g, x)$ is used. Betweenness centrality of a node measures the potential to mediate flows of resources or information between other nodes, and closeness centrality of a node measures the distances by efficient paths from the node to all the other nodes.

Fig. 3 illustrates the different behaviors of the two centrality measures in a certain types of graphs. In (A), centrality of $a$ is expected to be greater than that of $b$, since the node a make connection of two disconnected subgraphs, while the node $b$ is related with only single subgraph. Although both centralities of a are greater than those of $b$, betweenness centralities of $a$ and $b$ distinguish their social roles dramatically. On the other hand, in (B), closeness centralities can discriminate the social roles rather than betweenness centralities. In this case, related nodes with $c$ are more central than nodes related with $\mathrm{d}$. To assign a score $\mathcal{S}(g)$ of a gene $g$, we need to construct a temporary graph which is a subgraph of the most compatible reference graph $G_{M}$, whose nodes are set of genes in the pathway graph $G_{P}$
(A)

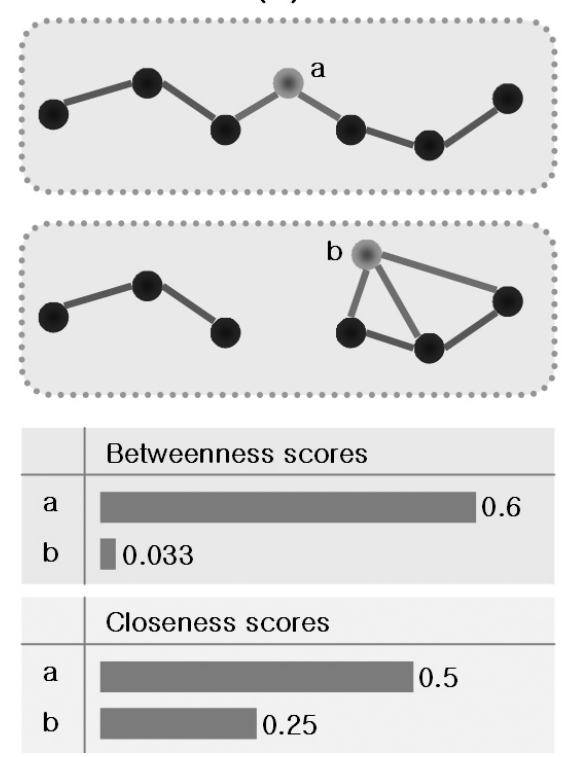

(B)

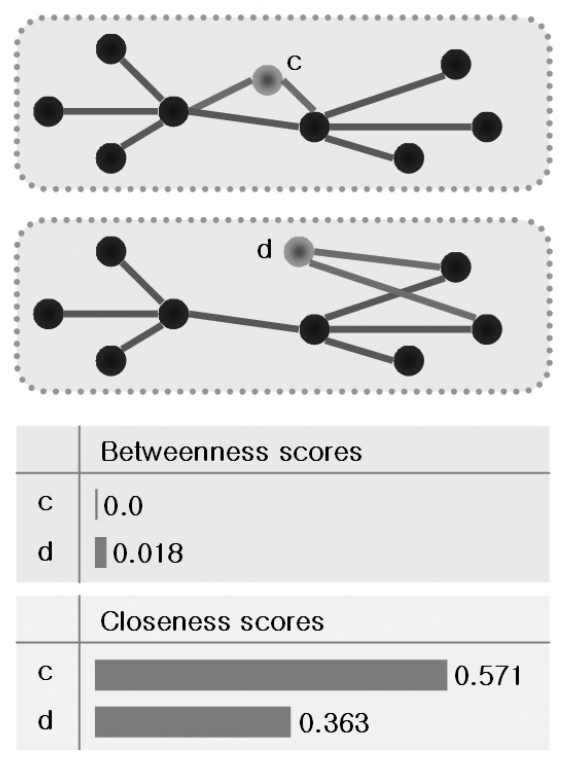

Fig. 3. Distribution of scores of specific nodes in certain types of graphs. In (A), betweenness scores of $a$ and $b$ distinguish social roles of $a$ and $b$ dramatically, while closeness scores discriminates social roles of $c$ and $d$ in $(B)$. 
plus a gene $g$. We define two scoring systems by normalized betweenness and closeness centrality measures of $g$ in the temporary graph. We call them the betweenness scoring system and closeness scoring system, respectively.

\section{Results and Discussion}

\section{Correlation between microarray data and path- way knowledge}

The basic assumption of PathPlus is that gene expression profile reflects pathway structure and pathway structure affects gene expression profile. We defined two 'expressional homogeneity' indices, co-degree and similarity, between a pair of genes.

Co-degree between two genes in a microarray geneexpression dataset is defined as the number of conditions in which both are differentially expressed. Similarity between two genes is defined as the absolute value of the Pearson correlation between the two gene profile vectors. Two genes directly connected in a pathway graph are expected to have higher homogeneity indices than those non-connected.

We further defined three more measures, $E(P, M)=$ the average of the homogeneity (i.e. co-degree or similarity) indices of all directly connected pairs in a pathway graph $P, N(P, M)=$ the average of the homogeneity (i.e. co-degree or similarity) indices of all pairs that are not directly connected in a pathway graph $P$, and $R(M)$ $=$ the average of homogeneity indices of all gene pairs in a microarray dataset $M$.

If the basic assumption of PathPlus holds, we expect that the inequality $E(P, M)>N(P, M)>R(M)$ should be satisfied. Fig. 4 demonstrates the distributions of the average homogeneity indices of directly connected and non-directly-connected gene pairs for selected pathways and those of all gene pairs from the whole microarray dataset. Because it is observed that the inequality holds, it is suggested that microarray gene-expression profile reflects biological pathway structures and vice versa. These measures were only introduced to show the correlation between microarray data and pathway knowledge.

\section{Performance evaluation of scoring systems}

PathPlus assigns a score for each candidate gene. We evaluated the reliability of the scoring system. To measure the reliability of scoring system or performance of model, we introduce a performance function, $f(k)=M(k) / n \cdot 100$ $(\%)$, where $M(k)$ is the number of genes that included in the original pathway among top $\mathrm{k}$ ranked genes, and $\mathrm{n}$ is the number of genes in the pathway, and $k$ runs from 0 to $N$, the number of candidate genes. $(N=6,153$ in this study).

Fig. 5 shows performance functions of each scoring systems in each graph-construction methods. It also
(A)

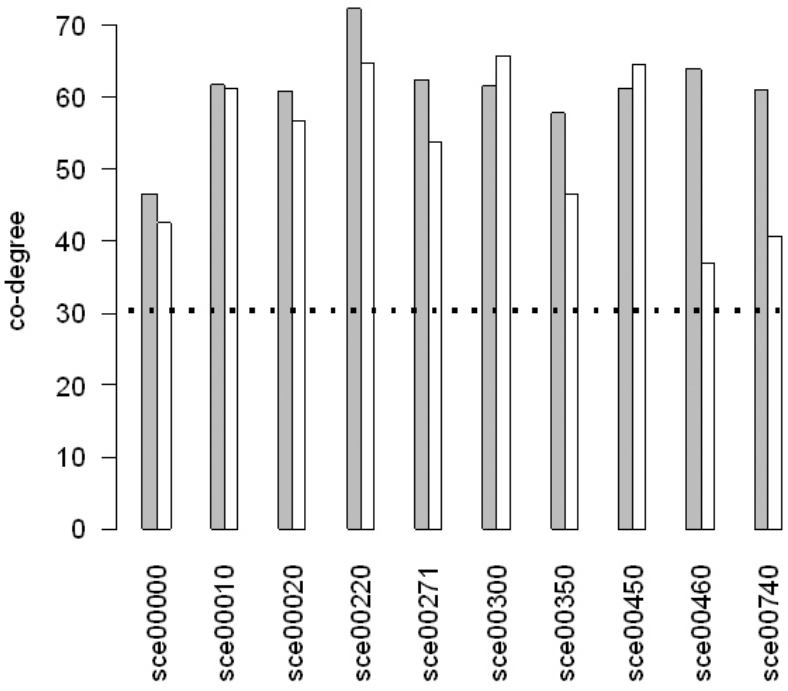

(B)

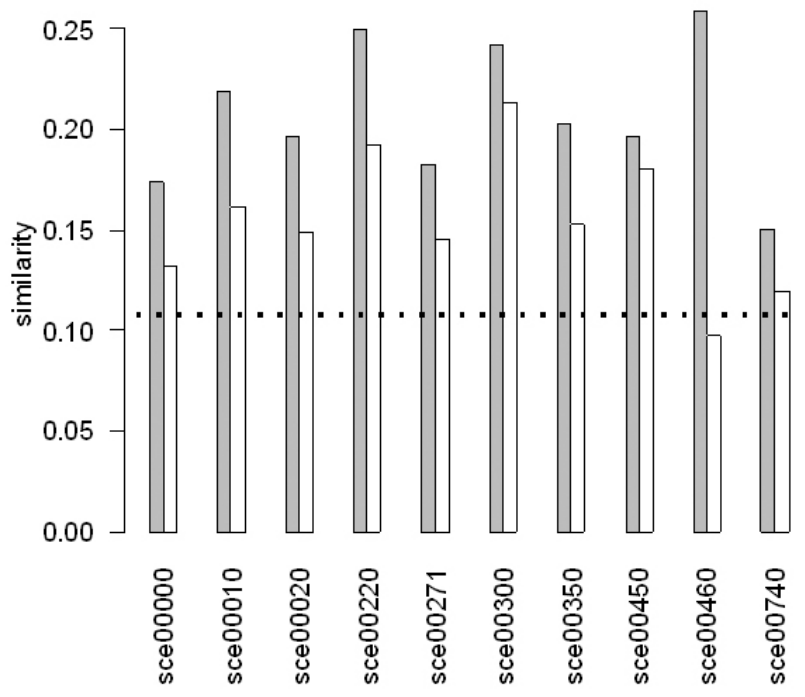

Fig. 4. Correlation between microarray data and knowledge about selected pathways. Both (A) Co-degree and (B) similarity of connected gene pairs (i.e. the gray bars) show higher values than non-connected ones (i.e. the white bars) for random selected 10 pathways. Overall results are similar to this result. The dotted horizontal lines represent the mean homogeneity values of the whole gene pairs. 
(A)

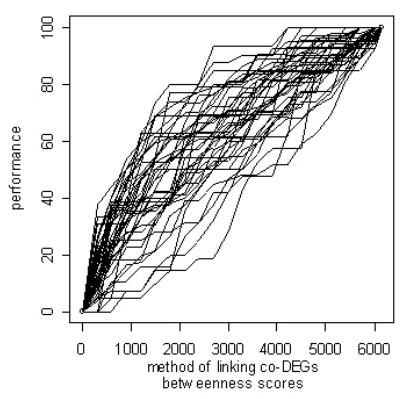

(E)

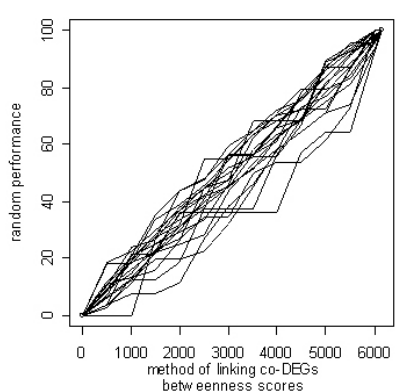

(B)

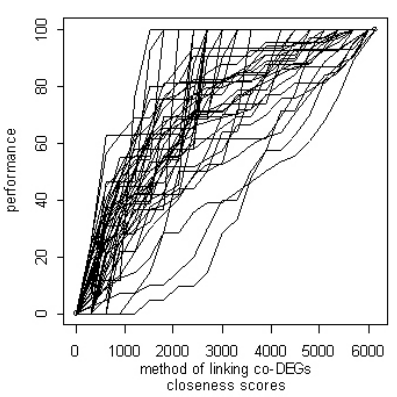

(F)

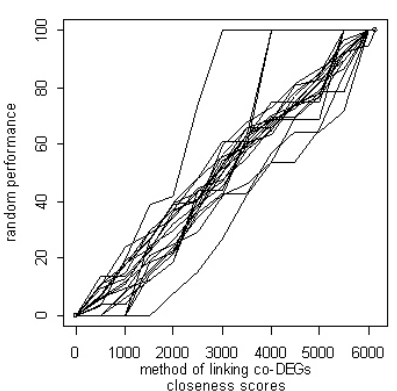

(C)

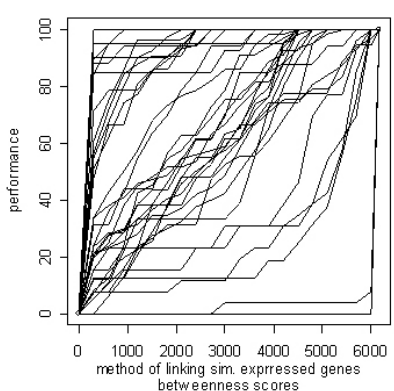

(G)

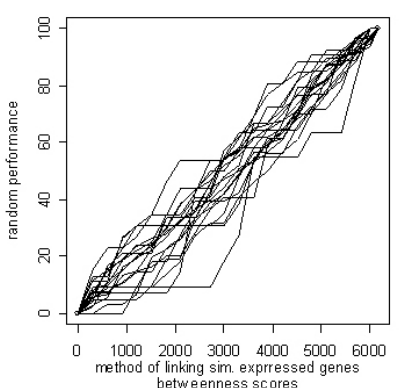

(D)

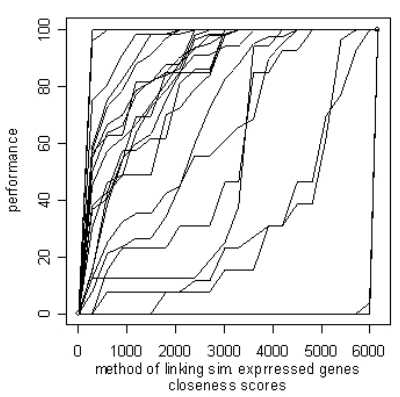

(H)

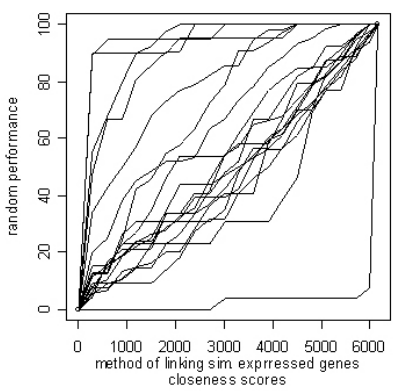

Fig. 5. Performance functions of pathways. (A), (B), (E), (F) use reference graph constructed by linking co-DEGs, (C), (D), (G), $(\mathrm{H})$ use method of linking similarly expressed genes. The first and third columns applied by betweenness scoring system, and the remains are applied by closeness one. The upper part give performances of actual pathways and the lower part show performances of randomly constructed pathways.

provided the performance of randomly constructed pathways.

Fig. $5(A),(B),(E)$ and $(F)$ are performance functions of pathways using the reference graph constructed by the method of linking co-DEGs, while (C), (D), (G) and $(H)$ are using the method of linking similarly expressed genes. The $(A),(C),(E)$ and $(G)$ are applied by the betweenness scoring system, and the remaining part $(B)$, (D), $(F)$ and $(H)$ are applied by the closeness scoring system. The upper part (A), (B), (C) and (D) show performances of actual pathways, but the lower part $(E)$, $(F),(G)$ and $(H)$ represent performances of randomly constructed pathways. As expected, performances functions of randomly constructed pathways are roughly located on the diagonal lines connecting origin and top-right point $(N, 100)$. The curves of actual performances are located in the upper triangular parts in general, so we expect that two scoring systems make quite good results of our purpose. Betweenness scoring systems give more stable performance with the reference graph constructed by linking co-DEGs. The reference graph obtained by the method of linking similarly expressed genes serves not-so-good performance scoring systems. These poor results maybe related to the similarity system (the absolute value of Pearson correlation coefficient) of two genes. Pearson correlation usually ig- nores the local similarity, i.e., it may assign low similarity to pair of genes which are highly co-expressed only in the small number of experimental conditions. The linking co-DEGs methods overcome the locality problem in some sense.

\section{Validation of the model via extrinsic information}

The candidate gene list has been obtained by applying our model to each pathway. Since we have two scoring systems and also two methods to construct reference graphs, each pathway has 4 lists of candidates. Table 1 and 2 show the top-ranked genes with respect to the pathways: "glycolysis / gluconegenesis" and "TCA cycle". These two pathways are selected due to their biological significances and performances. In these tables, we find that ranks of gene list from different scoring systems are quite compatible each other, but different types of constructing reference graph create inconsistent lists of candidates. Each table consists of two lists classified by its methods of graph construction, and in each part, sums of ranks $(r)$, ranks in betweenness scoring system $(r b)$, ranks in closeness scoring system $(r c)$ and grades are examined. The grades are assigned by the association with amiGO annotation and standard biological references. The grade " $A$ " is assigned to 
Genomics \& Informatics Vol. 6(4) 202-209, December 2008

Table 1. Candidate genes to extend pathway: Glycosis/ Gluconeogenesis

\begin{tabular}{|c|c|c|c|c|c|c|c|c|c|}
\hline \multicolumn{5}{|c|}{ Linking co-DEGs } & \multicolumn{5}{|c|}{ Linking similarly expressed genes } \\
\hline ORF & r & $\mathrm{rb}$ & rc & Grade & ORF & $r$ & $\mathrm{rb}$ & rc & Grade \\
\hline YFL014W & 3 & 2 & 1 & B & YBR019C & 2 & 1 & 1 & A \\
\hline YGL255W & 5 & 3 & 2 & C & YMR323W & 5 & 3 & 2 & B \\
\hline YML128C & 7 & 4 & 3 & $?$ & YLR377C & 6 & 2 & 4 & A \\
\hline YBR296C & 11 & 7 & 4 & $?$ & YKR097W & 7 & 4 & 3 & A \\
\hline YGL117W & 13 & 8 & 5 & ? & YPL017C & 11 & 5 & 6 & B \\
\hline YLR303W & 13 & 5 & 8 & B & YGL256W & 12 & 7 & 5 & B \\
\hline YFR053C & 14 & 1 & 13 & A & YAL054C & 13 & 6 & 7 & A \\
\hline YHR136C & 15 & 9 & 6 & $\mathrm{~B}$ & YDR050C & 18 & 10 & 8 & $A$ \\
\hline YKL096W & 17 & 10 & 7 & B & YDR045C & 19 & 9 & 10 & C \\
\hline YDL037C & 23 & 6 & 17 & $?$ & YJR141W & 19 & 8 & 11 & $?$ \\
\hline YCL018W & 27 & 11 & 16 & B & YDR380W & 20 & 11 & 9 & A \\
\hline YBR147W & 29 & 14 & 15 & $?$ & YPL146C & 25 & 13 & 12 & $?$ \\
\hline YGR110W & 33 & 12 & 21 & $?$ & YNL001W & 26 & 12 & 14 & $?$ \\
\hline YML123C & 33 & 24 & 9 & A & YMR233W & 34 & 14 & 20 & $?$ \\
\hline YGR035C & 35 & 15 & 20 & $?$ & YJL068C & 37 & 20 & 17 & B \\
\hline YMR094W & 35 & 25 & 10 & C & YCR095C & 40 & 17 & 23 & $?$ \\
\hline YMR096W & 37 & 26 & 11 & C & YLR336C & 42 & 23 & 19 & $?$ \\
\hline YNL160W & 39 & 27 & 12 & A & YJR003C & 48 & 30 & 18 & $?$ \\
\hline YNR058W & 48 & 13 & 35 & C & YLR287C & 50 & 21 & 29 & $?$ \\
\hline YBR047W & 50 & 36 & 14 & ? & YPR169W & 50 & 18 & 32 & $?$ \\
\hline
\end{tabular}

In the table, $r$ is for sums of ranks, rb for betweenness ranks, $r c$ for closeness ranks. The grades are assigned by the association with amiGO annotation. The grade "A" is for genes that clearly associated with the pathway, "B" is for genes that have equivocal interpretation, "C" is for genes without association and "?" is for genes with unknown functional annotation.

Table 2. Candidate genes to extend pathway: TCA cycle

\begin{tabular}{|c|c|c|c|c|c|c|c|c|c|}
\hline \multicolumn{5}{|c|}{ Linking co-DEGs } & \multicolumn{5}{|c|}{ Linking similarly expressed genes } \\
\hline ORF & $r$ & $\mathrm{rb}$ & rc & Grade & ORF & $r$ & $\mathrm{rb}$ & rc & Grade \\
\hline YBR296C & 2 & 1 & 1 & A & YJL045W & 2 & 1 & 1 & A \\
\hline YFL014W & 4 & 2 & 2 & B & YKR097W & 5 & 2 & 3 & A \\
\hline YGL255W & 6 & 3 & 3 & C & YMR323W & 5 & 3 & 2 & A \\
\hline YML128C & 8 & 4 & 4 & ? & YDR380W & 9 & 4 & 5 & A \\
\hline YIR039C & 12 & 5 & 7 & B & YDR148C & 10 & 6 & 4 & $?$ \\
\hline YGL117W & 15 & 9 & 6 & $?$ & YPL017C & 11 & 5 & 6 & B \\
\hline YBR072W & 17 & 7 & 10 & B & YHR196W & 18 & 8 & 10 & C \\
\hline YLR303W & 19 & 10 & 9 & C & YDR299W & 24 & 10 & 14 & $?$ \\
\hline YCL040W & 21 & 16 & 5 & A & YPL146C & 24 & 13 & 11 & $?$ \\
\hline YJL159W & 25 & 17 & 8 & C & YGR128C & 26 & 11 & 15 & B \\
\hline YOR383C & 33 & 8 & 25 & $?$ & YJR041C & 26 & 7 & 19 & B \\
\hline YPR167C & 34 & 6 & 28 & C & YJR003C & 27 & 9 & 18 & $?$ \\
\hline YFR053C & 35 & 22 & 13 & $A$ & YLR051C & 33 & 12 & 21 & $?$ \\
\hline YKL096W & 38 & 21 & 17 & $C$ & YJL068C & 38 & 21 & 17 & B \\
\hline YJL116C & 39 & 24 & 15 & $?$ & YLR022C & 38 & 18 & 20 & $?$ \\
\hline YCL018W & 45 & 12 & 33 & C & YPL210C & 40 & 16 & 24 & C \\
\hline YJL079C & 47 & 33 & 14 & $?$ & YBR142W & 43 & 30 & 13 & B \\
\hline YMR276W & 47 & 26 & 21 & C & YLR435W & 43 & 20 & 23 & $?$ \\
\hline YOR382W & 47 & 23 & 24 & $?$ & YHR197W & 45 & 14 & 31 & $?$ \\
\hline YJR028W & 50 & 34 & 16 & C & YBR242W & 50 & 23 & 27 & $?$ \\
\hline
\end{tabular}

In the table, $r$ is for sums of ranks, rb for betweenness ranks, $r c$ for closeness ranks. The grades are assigned by the association with amiGO annotation. The grade " $A$ " is for genes that clearly associated with the pathway, "B" is for genes that have equivocal interpretation, "C" is for genes without association and "?" is for genes with unknown functional annotation. 
genes that are clearly associated with the pathway, "B" is genes with equivocal interpretation, " $C$ " is genes without association and "?" is for the case of unknown functional annotation. All grade is endowed with subjectivity.

Let us look into the candidates for "glycolysis / gluconeogenesis"pathway. A few candidates are annotated a general or vital cellular function or component that we cannot easily judge the relevance to the pathway (YFL014W, YML128C, YHR136C, YKL096W and YMR094W). $A$ few others are not revealed about their functions (YBR296C, YGL117W, YDL037C, YGR110W, YBR147W and YGR035C). And some genes suggestive of strong relation with the pathway also have been found (YFR053C, YML123C, YNL160W and YBR296C). YFR053C functions as a hexokinase, which is one of the key enzymes of glycolysis but has been omitted from pathway graph because the gene ID has not been served by KEGG database (E.C 2.7.1.1. in glycolysis / gluconeogenesis pathway data from KEGG corresponds to it). YBR296C and YML123C are genes whose products function as phosphate transporters-glycolysis process is intrinsically linked with citric acid cycle, which is subsequently linked with oxidative phosphorylation. Therefore the extension of glycolysis / gluconeogenesis pathway toward oxidative phosphorylation via phosphate transporter can be sufficiently persuasive.

The next candidate list is the one for extension of citric acid cycle. This pathway is historically well known, and is considered to be centrally located within a metabolic pathway network. (Jeong et al., 2001) Hence almost every gene might be traced to its relation to citric acid cycle within a few connections.

Nevertheless, in the list are some genes that draw our attention (YLR303W, YCL040W, YCL018W, YOL151W, YCL030C, YDL022W, YFR053C and YJL116C). YLR303W deals with serine metabolism, which is initiated from a precursor of citric acid cycle, 3-phosphoglycerate. YCL040W is a gene with glucokinase activity that is essential in glycolysis/glucokinase pathway, which, as we mentioned above, is closely lined with citric acid cycle. YJL116C is a gene for mitochondrion organization and biogenesis and citric acid cycle occurs within mitochondrion. YOL151W is known to have oxidoreductase activity, which is crucial for oxidative phosphorylation- the very next step of citric acid cycle for energy generation. YDL022W is related to NAD activity, which plays a key role in citric acid cycle. Similarly, others participate in or are related to citric acid cycle; YCL018W is related to the cycle through malate metalbolism, YCL020C through histidine metabolism and YFR053C through fructose metabolism.

\section{Conclusions}

In this paper, we made an attempt to extend an established pathway through microarray expression data. To obtain this goal, we transformed knowledge about pathways and microarray expression data, respectively, into graph structures, and analyzed two graphs with a scoring system we invented based on social network theory. We also validated the reliability of the scoring system by computing prediction powers. Then final result of candidate lists has been presented and has been examined through another source of biological data, amiGO annotation.

\section{References}

Bogatti, S.P., and Everett, M.G. (1997). Network analysis of 2-mode data. Social Networks 19, 243-269.

Chartrand, G., Kubicki, G., and Schultz, M. (1988). Graph similarity and distance in graphs. Aequationes Math. 55, 129-145.

Dudoit, S., Yang, Y.H., Gallow, M.J., and Speed, T.P. (2002). Statistical methods for identifying differentially expressed genes in replicated cDNA microarray experiments. Statistica Sinica. 12, 111-139.

Faust, K. (1997). Centrality in affiliation networks. Social Networks 19, 157-191.

Friedman, N., Linial, M., Nachman, I., and Pe'er, D. (2000). Using Bayesian Network to Analyze Expression Data. Journal of Computational Biology 7, 601-620.

Hughes, T.R., Marton, M.J., Jones, A.R., Roberts, C.J., Stoughton, R., Armour, C.D., Bennett, H.A., Coffey, E., Dai, H., He, Y.D., Kidd, M.J., King, A.M., Meyer, M.R., Slade, D., Lum, P.Y., Stepaniants, S.B., Shoemarker, D.D., Gachotte, D., Chakraburtty, K., Simon, J., Bard, M., and Friend, S.H. (2000). Functional discovery via a compendium of expression profiles. Cell 102, 109-126.

Iyer, V.R., Eisen, M.B., Ross, D.T., Schuler, G., Moore, T., Lee, J.C., Trent, J.M., Staudt, L.M., Hudson, J. Jr., Boguski, M.S., Lashkari, D., Shalon, D., Botstein, D., and Brown, P.O. (1999). The transcriptional program in the response of human fibroblasts to serum. Science 283, 83-87.

Jeong, H., Mason, S.P., Barabasi, A.L., and Oltvai, Z.N. (2001). Lethality and centrality in protein networks. Nature 6833, 41-2.

Kanehisa, M. (1996). Toward pathway engineering: a new database of genetic and molecular pathways. Science \& Technology Japan 59, 34-38.

Kharchenko, P., Vitkuo, D., and Church, G.M. (2004). Filling gaps in a metabolic networks using expression information. Bioinformatics 20 (Suppl. 1), i178-i185.

Tusher, V.G., Tibshirani, R., and Chu, G. (2001). Significance analysis of microarrays applied to the ionizing radiation response. Proc. Natl. Acad. Sci. USA. 98, 5116-5121.

Yamanishi, Y., Vert, J.P., Nakaya, A., and Kanehisa, M. (2004). Protein network inference from multiple genomic data: a supervised approach. Bioinformatics 20 (Supple. 1), i363-i370. 